\title{
Evolução de parâmetros antropométricos em portadores do vírus da Imunodeficiência Humana ou com Síndrome da Imunodeficiência Adquirida: um estudo prospectivo'
}

\author{
Changes in the anthropometric parameters of \\ patients with the Human Immunodeficiency \\ virus or Acquired Immunodeficiency \\ Syndrome: a prospective study
}

Maíra Ladeia Rodrigues CURTI²

Luara Bellinghausen ALMEIDA²

Patrícia Constante JAIME²

RES U M O

\section{Objetivo}

Avaliar o estado nutricional e a evolução de parâmetros antropométricos para alterações morfológicas em pacientes vivendo com o vírus da Imunodeficiência Humana ou com Síndrome da Imunodeficiência Adquirida em uso de terapia antirretroviral de alta atividade.

\section{Métodos}

Trata-se de estudo de coorte prospectiva com duração de 12 meses, envolvendo indivíduos adultos, de ambos os sexos, em terapia antirretroviral recém introduzida. Os indicadores antropométricos estudados foram índice de massa corporal, circunferência de cintura, dobras cutâneas subescapular, biciptal e triciptal, avaliados com intervalos de três meses, totalizando 4 medidas do tempo. Variáveis foram descritas segundo mediana e percentis 25 e 75 e analisadas por ANOVA para medidas repetidas.

\section{Resultados}

A população estudada foi composta por 53 indivíduos, a maioria do sexo masculino (81\%), entre 30 e 39 anos. Apenas a dobra cutânea subescapular apresentou significante variação no tempo $(T 1=13,7$ vs $T 4=16,0$; $p<0,001)$, apontando para lipo-hipertrofia dorso-cervical.

1 Pesquisa financiada pela Fundação de Amparo à Pesquisa do Estado de São Paulo (processo no 04/12462-0) e Bolsa de Iniciação Científica para M.L.R. CURTI (processo no 05/50948-4).

2 Universidade de São Paulo, Faculdade de Saúde Pública, Departamento de Nutrição. Av. Dr. Arnaldo, 715, Cerqueira Cesar, 01246-904, São Paulo, SP, Brasil. Correspondência para/Correspondence to: P.C. JAIME. E-mail: <constant@usp.br>. 
58 | M.L.R. CURTI et al.

\section{Conclusão}

Os achados deste estudo, embora limitados, direcionam para a necessidade de vigilância de parâmetros antropométricos associados a alterações morfológicas, em especial, aqueles usados no diagnóstico de acúmulo de gordura abdominal e dorso-cervical.

Termos de indexação: Antropometria. Gordura corporal. Estado nutricional. Síndrome da Imunodeficiência Adquirida. Lipodistrofia.

\section{A B S T R A C T}

\section{Objective}

The objective of this study was to assess the nutritional status and changes in the anthropometric indicators of patients with the human Immunodeficiency virus or acquired immunodeficiency syndrome using the highly active antiretroviral therapy.

\section{Methods}

This is a 12-month prospective cohort study of adult males and females who recently started antiretroviral therapy. The anthropometric indicators studied were body mass index, waist circumference and subscapular, biceps and triceps skinfold thicknesses, taken 4 times during the year in 3-month intervals. The variables were described according to medians and 25 and 75 percentiles and analyzed by ANOVA for repeated measurements.

\section{Results}

The studied population consisted of 53 patients, mostly males (81\%) aged 30 to 39 years. Only subscapular skinfold thickness changed significantly over time (T1=13.70 vs $T 4=16.00, p<0.001)$, indicating cervical lipohypertrophy (buffalo hump).

\section{Conclusion}

The findings of this study, although limited, show the need to monitor anthropometric parameters associated with morphological changes, especially those used in the diagnosis of abdominal and dorsocervical fat accumulation.

Indexing terms: Anthropometry. Body fat. Acquired Immunodeficiency Syndrome. Nutritional status. Lipodystrophy.

\section{N T R O D U ÇÃ O}

Segundo estimativas da Joint United Nations Programme on HIVIAIDS', aproximadamente 39,6 milhões de pessoas vivem com HIV/ Aids em todo o mundo. No Brasil, estima-se haver 620 mil indivíduos infectados pelo HIV, sendo que o município de São Paulo concentra o maior número absoluto de indivíduos vivendo com HIV/Aids no País ${ }^{2}$. A partir de 1996, adotou-se a política de distribuição gratuita e universal de medicação antirretroviral no Sistema Único de Saúde brasileiro, sendo o Brasil um dos primeiros países em desenvolvimento a adotá-la3.

A terapia antirretroviral de alta atividade causou um profundo impacto na história da infecção pelo HIV, com drástica diminuição da mortalidade e da ocorrência de infecções oportunistas e subsequente aumento da sobrevida dos portadores ${ }^{4,5}$. No Brasil, a intensa queda da mortalidade por Aids evidencia o atual caráter crônico e o controle da evolução da doença ${ }^{3}$.

Concomitantemente a esses avanços terapêuticos, a literatura descreve o surgimento de alterações metabólicas e morfológicas decorrentes do uso da terapia antirretroviral de alta atividade, que compõem a chamada síndrome lipodistrófica do HIV ${ }^{6}$. Dentre as alterações morfológicas estão a lipoatrofia em regiões periféricas do corpo, expressa por perda de gordura na face, nos membros e nas nádegas ${ }^{7,9}$, além de proeminência muscular e venosa relativa ${ }^{7}$; e a lipo-hipertrofia em regiões centrais do corpo, tais como aumento de gordura em região abdominal4,7,8,9, gibosidade dorsal e aumento da mama em mulheres ${ }^{4,7}$. 
Se, anteriormente, os pacientes com Aids eram identificados e estigmatizados pela presença de sinais visuais da doença, tais como o sarcoma de Kaposi, atualmente as alterações morfológicas constroem um novo estereótipo da doença, refletindo na qualidade de vida dos indivíduos acometidos9,10,11. Outra preocupação no cuidado à saúde mental e física desses pacientes seria o risco potencial de doenças aterogênicas, já que o acúmulo de gordura central está associado à síndrome metabólica e ao maior risco para doenças crônicas não transmissíveis ${ }^{4,12,13}$. Assim, a investigação e o diagnóstico precoce das alterações morfológicas em indivíduos infectados pelo vírus HIV em uso de terapia antirretroviral de alta atividade, são imperativos para a identificação de riscos e o contínuo alcance dos objetivos de assistência a longo prazo nesta população ${ }^{14-16}$.

O objetivo deste estudo foi avaliar o estado nutricional e a evolução de parâmetros antropométricos para alterações morfológicas em pacientes vivendo com HIV/Aids em uso de terapia antirretroviral de alta atividade recém introduzida.

\section{MÉ T O D O S}

Este estudo é uma coorte prospectiva com duração de 12 meses, envolvendo amostra de indivíduos adultos de ambos os sexos, usuários de um serviço de referência no tratamento de HIV/Aids no município de São Paulo.

Os critérios de inclusão adotados foram: paciente ter idade entre 20 e 59 anos e estar há, no máximo, um ano do início do estudo, em uso de terapia antirretroviral no esquema de terapia tripla, incluindo drogas com Inibidor da Transcriptase Reversa Análogo de Nucleosídeo (ITRN), Inibidor da Transcriptase Reversa não Análogo de Nucleosídeo (ITRNN) e Inibidor de Protease (IP). Os critérios de exclusão foram apresentar obesidade centrípeta e perda de gordura corporal periférica, idade menor que 18 e maior que 59 anos, estar gestante, ter se submetido à intervenção plástica que refletisse em alteração de peso e de distribuição de gordura, apresentar câncer, fazer uso de hipoglicemiante, hipolipemiante, corticosteróides e anabolizantes. Nesse serviço de referência são atendidos cerca de 4 mil pacientes, sendo identificados 2870 em uso de terapia antirretroviral na época de seleção da amostra. Destes, 84 atendiam aos critérios de inclusão e exclusão do estudo, dos quais 31 não aderiram ao protocolo, resultando, assim, na amostra de 53 indivíduos estudados.

Os indicadores antropométricos utilizados buscaram refletir o estado nutricional dos indivíduos e a deposição de gordura em áreas específicas do corpo. O exame antropométrico foi feito em quatro momentos: no $1^{\circ}$ mês (T1), no $4^{\circ}(\mathrm{T} 2)$, no $8^{\circ}$ (T3) e no $12^{\circ}$ segundo mês (T4). Todas as medições foram feitas especificamente para o presente estudo e conduzidas por nutricionista devidamente treinada pelo Laboratório de Avaliação Nutricional de Populações (LANPOP) da Faculdade de Saúde Pública da Universidade de São Paulo.

Na avaliação do estado nutricional, o indicador utilizado foi o Índice de Massa Corporal (IMC). Para o cálculo do IMC peso e estatura foram mensurados aplicando-se as técnicas de medição antropométricas recomendadas ${ }^{17}$. Os participantes do estudo foram, então, classificados pelo seu estado nutricional em baixo peso (IMC $<18,5 \mathrm{~kg}$ / $\mathrm{m} 2$ ), eutróficos (IMC entre 18,5 e $25 \mathrm{~kg} / \mathrm{m}^{2}$ ), sobrepeso (IMC entre 25 e $30 \mathrm{~kg} / \mathrm{m}^{2}$ ) e obesos (IMC> $30 \mathrm{~kg} / \mathrm{m}^{2}$ ), segundo critérios da Organização Mundial da Saúde ${ }^{17}$.

O acúmulo de gordura foi avaliado pela circunferência da cintura e pela dobra cutânea subescapular. As dobras cutâneas de bíceps e tríceps foram os indicadores antropométricos utilizados na avaliação da perda de gordura periférica. Essas medidas foram repetidas três vezes em cada momento de coleta, usando-se a média para fazer as análises estatísticas. Aplicaram-se as técnicas de medição antropométricas recomendadas por Lohman et al. ${ }^{18}$.

Em adição aos indicadores antropométricos, foram investigadas características sócio-demográficas (sexo, idade, escolaridade e renda) 
60 | M.L.R. CURTI et al.

e clínicas dos pacientes (tempo de infecção pelo vírus da Aids, tempo de uso de terapia antirretroviral de alta atividade, determinação do número de linfócitos T $\left(D 4^{+}\right)$. Esses dados foram obtidos pelos registros nos prontuários médicos e por questionário padronizado, aplicado por entrevistador treinado.

$\mathrm{Na}$ análise estatística, o conjunto de variáveis foi descrito segundo número e proporção de casos, por medidas de tendência central (mediana) e de dispersão (percentis 25 e 75), dada a não aderência à distribuição normal das variáveis estudadas, após a aplicação do teste de Kolmogorov-Smirnov.

Para analisar a evolução dos parâmetros antropométricos no tempo utilizou-se Análise de Variância (ANOVA) para medidas repetidas. A análise dos dados foi feita segundo o método Intention-to-treat (Intenção de tratar) por meio da técnica Last Outcome Measure Carried Forward (LOCF), na qual a última medida do indivíduo é considerada até a avaliação final da coorte. O nível de significância adotado para os testes estatísticos foi de $5 \%(p<0,05)$. Para as análises estatísticas foi utilizado o programa SAS System para Windows, versão 8.02 .

Este estudo foi aprovado (protocolo $\mathrm{n}^{\circ}$ 436/2005) pelo Comitê de Ética e Pesquisa do Hospital das Clínicas da Faculdade de Medicina e pelo Comitê de Ética da Faculdade de Saúde Pública, ambos da Universidade de São Paulo.

\section{RES U LT A D O S}

Avaliaram-se 53 indivíduos, sendo que houve perda de seguimento para 11 deles, totalizando 42 pacientes na última avaliação. Os pacientes estudados caracterizam-se, em sua maioria, por indivíduos do sexo masculino, com idades entre 30 e 39 anos, de escolaridade média e renda de até 3 salários-mínimos. Na distribuição da população segundo seu estado nutricional, avaliado pelo IMC, observa-se que a maioria foi classificada como eutrófica (71,7\%), sendo que a proporção de pacientes com sobrepeso superou a com baixo peso (Tabela 1).
Tabela 1. Distribuição da população de adultos portadores do vírus da Imunodeficiência Humana ou com Síndrome da Imunodeficiência Adquirida (HIV/AIDS), segundo características sócio-demográficas e estado nutricional de acordo com categorias do índice de massa corporal. São Paulo (SP), 2005.

\begin{tabular}{|c|c|c|}
\hline Variáveis & $n=53$ & $\% 100$ \\
\hline \multicolumn{3}{|l|}{ Sexo } \\
\hline Masculino & 43 & 81,13 \\
\hline Feminino & 10 & 18,87 \\
\hline \multicolumn{3}{|l|}{ Idade } \\
\hline $20-29$ anos & 12 & 22,64 \\
\hline 30 - 39 anos & 26 & 49,05 \\
\hline$\geq 40$ anos & 15 & 28,03 \\
\hline \multicolumn{3}{|l|}{ Escolaridade } \\
\hline Ensino fundamental & 14 & 26,40 \\
\hline Ensino médio & 21 & 39,62 \\
\hline Ensino superior & 18 & 33,97 \\
\hline \multicolumn{3}{|l|}{ Renda $(n=50)$} \\
\hline < 3 salários-mínimos & 22 & 44,00 \\
\hline $3<5$ salários-mínimos & 15 & 30,00 \\
\hline > 5 salários-mínimos & 13 & 26,00 \\
\hline \multicolumn{3}{|c|}{ Índice de massa corporal $\left(\mathrm{kg} / \mathrm{m}^{2}\right)$} \\
\hline Baixo peso $\left(<18,5 \mathrm{~kg} / \mathrm{m}^{2}\right)$ & 2 & 3,80 \\
\hline Eutrófico $\left(18,5-25 \mathrm{~kg} / \mathrm{m}^{2}\right)$ & 38 & 71,70 \\
\hline Sobrepeso $\left(25-30 \mathrm{~kg} / \mathrm{m}^{2}\right)$ & 13 & 24,50 \\
\hline Obesidade $\left(>30 \mathrm{~kg} / \mathrm{m}^{2}\right)$ & 0 & 0 \\
\hline
\end{tabular}

No tocante aos aspectos clínicos relacionados à infecção, o tempo de HIV/Aids caracteriza a amostra com recente conhecimento da doença (mediana de 1,5 ano). O mesmo observase sobre o tempo de uso de antirretrovirais (mediana de 8 meses). A contagem de linfócitos T-CD4+ é um dos parâmetros indicativos para o início da terapia antirretroviral em portadores de HIV e também para avaliar a resposta ao tratamento. O padrão desejável é acima de $350 / \mathrm{mm}^{3}$. Observou-se, nesta população, mediana de $300 / \mathrm{mm}^{3}$, sinalizando recente diagnóstico da doença e início da terapia antirretroviral de alta atividade (Tabela 2).

$\mathrm{Na}$ avaliação evolutiva dos indicadores antropométricos (fez-se análise de medidas repetidas) nota-se discreta diminuiç̧ão do IMC ao longo do tempo ( $T 1=23,29$ versus $T 4=22,93)$, enquanto que a circunferência da cintura aumentou, aproximadamente, $2 \mathrm{~cm}(T 1=83,2$ versus $T 4=85,4 \mathrm{~cm})$. 
Contudo, essas variações não apresentaram significância estatística. Para os indicadores de lipoatrofia periférica, a dobra cutânea do bíceps evoluiu sem alterações, quando o esperado é que houvesse uma diminuição dos valores. A dobra cutânea triciptal sofreu um discreto aumento, mas sem significância estatística; a dobra cutânea subescapular foi a única variável que apresentou significante aumento $(p<0,001)$, o que aponta para uma tendência de lipo-hipertrofia na região dorso-cervical (Tabela 3).

\section{I S C U S S Ã O}

A avaliação do estado nutricional dos indivíduos vivendo com Aids tem merecido importante destaque, por sua inter-relação com o estado de saúde e com a evolução da doença. Na era inicial da epidemia, anterior à introdução da terapia antirretroviral de alta atividade, os doentes eram intensamente acometidos pela desnutrição e por deficiências nutricionais ${ }^{19,20}$. Atualmente, se depara com um novo quadro, em que o caráter crônico da infecção pelo HIV coexiste com os problemas associados ao excesso de peso, ao acúmulo de gordura e à maior predisposição para síndrome metabólica ${ }^{8,13,14}$.

Embora ainda não se tenha padronizado um protocolo específico para a avaliação nutricional nesses pacientes, o monitoramento da evolução de parâmetros antropométricos, como peso,

Tabela 2. Características clínicas de adultos portadores do vírus da Imunodeficiência Humana ou com Síndrome da Imunodeficiência Adquirida (HIV/AIDS). São Paulo (SP), 2005.

\begin{tabular}{lccc}
\hline Variáveis clínicas & Mediana $(\mathrm{n}=53)$ & $P 25$ & $P 75$ \\
\hline Contagem de linfócitos & 300,00 & 186,00 & 361,00 \\
T-CD4 $+\left(\mathrm{mm}^{3}\right)$ & & & \\
Tempo de HIV/Aids (anos) & 1,50 & 1,00 & 4,00 \\
Tempo TARV (meses) & 8,00 & 5,00 & 12,00 \\
\hline
\end{tabular}

TARV: Terapia antirretroviral de alta atividade.

Tabela 3. Evolução dos parâmetros antropométricos de adultos portadores do vírus da Imunodeficiência Humana ou com Síndrome da Imunodeficiência Adquirida (HIV/AIDS), nos quatro momentos de avaliação. São Paulo (SP), 2006.

\begin{tabular}{|c|c|c|c|c|c|c|}
\hline \multirow{2}{*}{ Variável } & & \multicolumn{4}{|c|}{ Momento de avaliação } & \multirow{2}{*}{$\begin{array}{c}\text { Comparação entre os } \\
\text { tempos } p^{*}\end{array}$} \\
\hline & & T1 $(n=53)$ & $T 2(n=53)$ & T3 $(n=49)$ & $\mathrm{T} 4(\mathrm{n}=42)$ & \\
\hline \multirow{3}{*}{ Índice de massa corporal $\left(\mathrm{kg} / \mathrm{m}^{2}\right)$} & Mediana & 23,29 & 23,22 & 22,73 & 22,93 & \multirow{3}{*}{0,791} \\
\hline & P25 & 21,36 & 21,42 & 21,68 & 21,49 & \\
\hline & P75 & 25,00 & 25,23 & 24,58 & 24,84 & \\
\hline \multirow{3}{*}{ Circunferência de cintura (cm) } & Mediana & 83,20 & 84,60 & 84,60 & 85,40 & \multirow{3}{*}{0,105} \\
\hline & P25 & 79,30 & 79,70 & 80,00 & 79,60 & \\
\hline & P75 & 89,40 & 90,00 & 89,20 & 88,60 & \\
\hline \multirow{3}{*}{ Dobra cutânea bicipital (mm) } & Mediana & 4,00 & 4,50 & 4,60 & 4,00 & \multirow{3}{*}{0,067} \\
\hline & P25 & 3,00 & 3,50 & 3,30 & 4,00 & \\
\hline & P75 & 6,50 & 8,30 & 8,30 & 6,60 & \\
\hline \multirow{3}{*}{ Dobra cutânea tricipital (mm) } & Mediana & 8,30 & 9,60 & 9,60 & 9,30 & \multirow{3}{*}{0,670} \\
\hline & P25 & 6,50 & 6,30 & 6,30 & 7,00 & \\
\hline & P75 & 14,00 & 15,60 & 14,60 & 15,00 & \\
\hline \multirow{3}{*}{ Dobra cutânea subescapular (mm) } & Mediana & 13,70 & 15,00 & 16,60 & 16,00 & \multirow{3}{*}{$<0,001^{a}$} \\
\hline & P25 & 10,80 & 12,00 & 12,60 & 14,00 & \\
\hline & P75 & 17,50 & 19,30 & 20,60 & 20,00 & \\
\hline
\end{tabular}

* resultado das Análises de Variância para medidas repetidas usando o método ITT (Intention to Treat). $n=53$.

a diferença significante entre os tempos (teste de Perfil) $(\mathrm{T} 1 \neq \mathrm{T} 3, \mathrm{~T} 1 \neq \mathrm{T} 4)$. 
IMC, circunferência da cintura e dobras cutâneas; pode trazer informações valiosas para o diagnóstico precoce de alterações morfológicas secundárias à terapia antirretroviral.

Nesta população estudada, a proporção de indivíduos com sobrepeso (24,5\%) superou o baixo peso (2,8\%). A mediana de IMC no início do estudo foi de $23,3 \mathrm{~kg} / \mathrm{m}^{2}$, sendo que este valor não sofreu variação significante ao longo dos 12 meses de estudo, o que sinaliza evolução favorável para este parâmetro antropométrico. No entanto, apesar da reconhecida validade do IMC como indicador de risco de co-morbidades na população em geral ${ }^{17}$, e em pessoas vivendo com Aids ${ }^{21}$, ele é um parâmetro de avaliação nutricional insuficiente para a detecção de riscos e de indícios de redistribuição de gordura corporal.

Nesse sentido, avaliou-se a circunferência da cintura como indicador de acúmulo de gordura abdominal. Nota-se tendência de aumento dos valores medianos ao serem comparados os valores iniciais com os finais ( $T 1=83,2$ versus $T 4=85,4 \mathrm{~cm}$ ). Contudo, esta variação ao longo do tempo não apresentou significância estatística $(p=0,105)$, possivelmente em função do limitado número de casos estudados. A progressão continuada do acúmulo de gordura abdominal pode representar possível aumento de risco cardio-metabólico. Achados do estudo Fat Redistribution and Metabolic Change in HIV Infection (FRAM), realizado em 2005, mostraram prevalências de síndrome metabólica em 15,8\% dos pacientes em terapia retroviral de alta atividade, contra 3,2\% dos pacientes controles não portadores de HIV22.

Em relação às medidas de dobras cutâneas, elas são usualmente utilizadas como um método relativamente simples e não invasivo para a avaliação da distribuição do tecido adiposo subcutâneo ${ }^{18}$. Estudo de validação para medidas antropométricas em pessoas vivendo com HIV/AIDS, conduzido por Florindo et al..23, corrobora a validade das dobras cutâneas para a estimativa da gordura corporal total, de tronco e de membros nesta população específica. No presente estudo, observou-se que, após um ano de segui- mento, a dobra cutânea subescapular apresentou significante aumento, o que aponta para uma tendência de lipo-hipertrofia na região dorso-cervical. O acúmulo de gordura nesta região é um tipo de anormalidade morfológica da síndrome lipodistrófica associada ao HIV, e foi objetivamente confirmado por exame de absortometria por dupla emissão de raios $X$ (DEXA), em estudo realizado por Carr et al. ${ }^{24}$.

Observou-se que nem todos os pacientes estudados desenvolveram redistribuição de gordura, o que sustenta a hipótese de que a susceptibilidade às alterações morfológicas varia de acordo com fatores relacionados não somente à terapia antirretroviral24,25, mas também em função de fatores comportamentais, tais como práticas alimentares e atividade ${ }^{8,26}$.

Neste conjunto etiológico multifatorial, os tipos de medicamentos antirretrovirais em uso exerce importante influência na ocorrência das alterações morfológicas e metabólicas associadas à terapia ${ }^{27}$. Nesse contexto, destaca-se o advento farmacológico recente, que é marcado pelo empenho na introdução de drogas mais modernas e com menores efeitos potenciais para lipodistrofia. No presente estudo, não foi feito controle por tipo de droga antirretroviral utilizada pelo paciente, tendo sido considerado apenas o uso conjunto das três classes disponíveis - IP, ITRN e ITRNN, o que é uma reconhecida limitação do estudo.

Ainda, na análise da evolução dos parâmetros antropométricos não é possível destacar um possível viés associado ao tempo de seguimento dos participantes (12 meses), que pode ter sido insuficiente para ocorrência de alterações morfológicas. Por outro, é preciso reconhecer que a duração de uma coorte observacional é determinada pela viabilidade de manutenção dos participantes no estudo e pelos limites de operacionalização e de recursos da pesquisa.

Em conclusão, os achados deste estudo, embora limitados, direcionam para a necessidade de vigilância de parâmetros antropométricos associados a alterações morfológicas, em especial, aqueles usados no diagnóstico de acúmulo de gordura abdominal e dorso-cervical. 


\section{COLABORADORES}

M.L.R. CURTI e L.B. ALMEIDA participaram do planejamento do estudo, da coleta e da análise dos dados, da interpretação dos resultados e da redação do manuscrito. P.C. JAIME participou da concepção do estudo, coordenou o seu planejamento, supervisionou as etapas de coleta, análise e interpretação dos dados, assim como colaborou com a redação da versão final do manuscrito.

\section{REFER Ê N CIAS}

1. Joint United Nations Programme on HIV/AIDS (UNAIDS). 2006 Report on the global AIDS epidemic: a UNAIDS 10th anniversary special edition. Geneva: Unaids; 2006.

2. Brasil. Ministério da Saúde. Programa Nacional de DST e AIDS. 26a semanas epidemiológicas 2006. Bol Epidemiol AIDS/DST. 2006; 3(1):1-50.

3. Dourado I, Veras MASM, Barreira D, Brito AM. AIDS epidemic trends after the introduction of antiretroviral therapy in Brazil. Rev Saúde Pública. 2006; 40 (Supl):9-17. doi: 10.1590/s0034-8910 2006000800003.

4. Valente MM, Reis AF, Machado DM, Succi RCM, Chacra AR. Alterações metabólicas da síndrome lipodistrófica do HIV. Arq Bras Endocrinol Metabol. 2006; 49(6):871-81.

5. Li HY, Silva ACCMS, Santos SS. Síndrome lipodistrófica e HIV/Aids. J Bras Aids. 2002; 3(2):23-35.

6. Lumpkin M. FDA Public health advisory: reports of diabetes and hyperglycemia in patients receiving protease inhibitor for the treatment of human immunodeficiency virus (HIV). Washington (DC): US Government Printing Office; 1997.

7. Basílio de Oliveira CA, Valle HA, Serra M, Skowronski CSP. Lipodistrofia na AIDS. In: ATLAIDS Atlas de patologia da SIDA. São Paulo: Atheneu; 2005.

8. Santosa CP, Felipe $Y X$, Bragab PE, Ramosa D, Lima RO, Segurado AC. Self-perception of body changes in persons living with HIV/AIDS: prevalence and associated factors. AIDS 2005; 19(Supl 4): S14-S21. doi: 10.1590/s0034-89102220060005 0012.

9. Jaime PC, Florindo AA, Latorre MRDO, Segurado AAC. Central obesity and dietary intake in HIV/ AIDS patients. Rev Saúde Pública. 2006; 40(4): 634-40.

10. Power R, Tate HL, McGill SM, Taylor C. A qualitative study of the psychosocial implications of lipodystrophy syndrome on HIV positive individuals. Sex Transm Infect 2003; 79(2):137-41. doi: 10:1136/sti.79(2)137-41.

11. Burgoyne R, Collins E, Wagner C, Abbey S, Halman $M$, Nur $M$, et al. The relationship between lipodystrophy-associated body changes and measures of quality of life and mental health for HIV-positive adults. Qual Life Res. 2005; 14(4): 981-90. doi: 10.1007/s11136-004-2580-2.

12. World Health Organization. Diet, nutrition and the prevention of chronic diseases. Report of a joint WHO/FAO expert consultation. Geneva; 2003. WHO Technical Report Series, 916.

13. Gelato MC. Insulin and carbohydrate dysregulation. Clin Inf Dis. 2003; 36 (Suppl.2): 91-5. doi: 1058.48 38/2003/3607s2-0007.

14. Health KV, Hogg RS, Chan KJ, Harris M, Montessori $\mathrm{V}$, Oshaughnessy MV, et al. Lipodystrophyassociated morphological, cholesterol and triglyceride abnormalities in population-based HIV/ AIDS treatment database. AIDS 2001; 15(3):231-9.

15. Almeida LB, Jaime PC. Aspectos atuais sobre nutrição e AIDS na era da terapia antiretroviral de alta atividade. J Bras AIDS. 2006; 7(1):1-48.

16. Knox TA, Zafonte-Sanders M, Fields-Gardner C, Moen K, Johansen D, Paton N. Assessment of nutritional status, body composition, and human immunodeficiency virus-associated morphologic changes. Clin Infetc Dis. 2003 (Suppl 2):63-8. doi: 1058.4838/2003/3607s2-0003.

17. World Heath Organization. Physical status: the use and interpretation of anthropometry. Geneva: WHO; 1995 Technical Report Series, 854.

18. Lohman TG, Roche AF, Matorell R. Anthropometric standartization reference manual. Illinois: Human Kinetics Books; 1998. p.3-70.

19. Salomon J, De Truchis P, Melchior JC. Nutrition and HIV infection. Br J Nutr. 2002; 87(Suppl 1):111-9. doi: 10.1079/bjn2001464.

20. American Dietetic Association. Position of the American Dietetic Association and Dietitians of Canada: nutrition intervention in the care of persons with human immunodeficiency virus infection. J Am Diet Assoc. 2004; 104(9):1425-41. doi: 10.1016/j.jada.2004.07.012.

21. Meininger G, Hadigan C, Rietschel P, Grinspon S. Body-composition measurements as predictors of glucose and insulin abnormalities in HIV positive men. Am J Clin Nutr. 2002; 76(2):460-5.

22. Bacchetti P, Gripshover B, Grunfeld C, Heymsfield $\mathrm{S}$, McCreath $\mathrm{H}$, Osmond $\mathrm{D}$, et al. of fat redistribution and metabolic change in HIV infection (FRAM). fat distribution in men with HIV infection. J Acquir Immune Defic Syndr. 2005; 40(2):121-31. 
23. Florindo AA, Latorre MRDO, Santos ECM, Borelli A, Rocha MS, Segurado AAC. Validação de métodos de estimativas da gordura corporal em portadores do HIV/Aids. Rev Saúde Pública. 2004; 38 (5):643-9. doi: 10.1590/S0034-8910200400050 0005.

24. Carr A, Samaras K, Burton S, Freund J, Chisholm DJ, Cooper DA. A syndrome of peripheral lipodystrophy, hyperlipidaemia and insulin resistance in patients receiving HIV protease inhibitors. AIDS, 1998; 12(7):F51-F58.

25. Walli R, Herfort O, Michl G, Demant T. Treatment with protease inhibitors associated with peripheral insulin resistance and impaired oral glucose tolerance in HIV-1 infected patients. AIDS 1998; 12(15):F167-F173.

26. Lenhard JM, Croom DK, Weiel JE, Spaltenstein A. Dietary fat alters HIV protease inhibitor-induced mice. J Nutr. 2000; 130(9):2361-6.

27. Chen D, Misra A, Gard A. Lipodystrophy in Human Immunodeficiency virus-infected patients. J Clin Endocrinol Metab. 2002; 87(11):4845-56. doi: 10.1210/jc.2002-020794.

Recebido em: 10/3/2008

Versão final reapresentada em: 13/2/2009

Aprovado em: 15/6/2009 